\title{
COMMUNICATION
}

\section{Le fibrome odontogène central : à propos d'un cas}

\section{Belmehdi A, El Harti K, El Wady W}

Service de chirurgie orale, Faculté de médecine dentaire, Université Mohamed V, Rabat, Maroc

\section{Introduction}

Le fibrome odontogène central $(F O C)$ est une tumeur bénigne rare qui représente $0,1 \%$ de toutes les tumeurs odontogènes. Cliniquement, cette lésion se développe lentement et conduit à l'expansion corticale. Radiologiquement, la constatation la plus commune est radioclarté multiloculaire. Les lésions sont associées à la couronne d'une molaire, prémolaire ou incisive incluse et, dans certains cas, à la résorption ou le déplacement des racines $(1,2)$.

\section{Observation clinique}

II s'agit d'une patiente âgée de 15 ans, qui s'est présentée pour une tuméfaction dans la région postérieure maxillaire gauche évoluant progressivement depuis l'enfance.

La radiographie panoramique a montré une lésion radioclaire aux limites imprécises, englobant la 26 et la 27 incluse, refoulée en haut et en arrière. Un dentascan a été réalisé pour préciser les limites de la lésion.

Le traitement a été chirurgical par exérèse de la lésion. L'examen anatomopathologique a conclu à un fibrome odontogène central.

\section{Discussion}

Le FOC est une tumeur bénigne rare qui pourrait apparaître très similaire aux autres tumeurs odontogènes. Cependant, le fibrome odontogène est considéré comme un néoplasme distinct avec ses propres caractéristiques histopathologiques et cliniques le différenciant des autres tumeurs odontogéniques (1).

II dérive du mésenchyme odontogène et représente $9,4 \%$ de l'ensemble des tumeurs odontogènes. Cette tumeur est à prédominance féminine, diagnostiquée le plus souvent entre la deuxième et la troisième décennie, cependant, certains cas ont été rapportés entre 5 ans et 80 ans $(2,3)$.

Cliniquement, elle varie d'une tuméfaction asymptomatique à un aspect plus agressif provoquant des déplacements dentaires et des résorptions radiculaires.

\section{Conclusion}

Le fibrome odontogène central est une entité tumorale particulière dont les caractéristiques cliniques et radiologiques imposent un diagnostic différentiel avec d'autres lésions kystiques ou tumorales. Seul l'examen histologique permet un diagnostic de certitude.

Le traitement chirurgical conservateur par exérèse de la tumeur et l'instauration d'un suivi régulier permettent d'améliorer le pronostic de la lésion et de prévenir les récidives à long terme.

\section{Références}

1-Mosqueda-Taylor A, et al. Central odontogenic fibroma: New findings and report of a multicentric collaborative study. Oral Surg Oral Med Oral Pathol Oral Radiol Endod 2011;112:349-58.

2- El Harti K et al. Central odontogenic fibroma of the maxilla. Indian J Dent 2015;6:217-20.

3-Covani U et al. Central odontogenic fibroma: A case report. Med Oral Patol Oral Cir Bucal 2005;10:E154_7.

This is an Open Access article distributed under the terms of the Creative Commons Attribution License 4.0, which permits unrestricted use, distribution, and reproduction in any medium, provided the original work is properly cited. Article disponible sur le site http://www.stco-congres.org ou http://dx.dol.org/10.1051/stco/20166402004 